\title{
Non-targeted and targeted analysis of oxylipins in combination with charge-switch derivatization by ion mobility high-resolution mass spectrometry
}

\author{
Stefan Hellhake ${ }^{1} \cdot$ Sven W. Meckelmann ${ }^{2} \cdot$ Michael T. Empl $^{3} \cdot$ Kristina Rentmeister $^{2} \cdot$ Walter Wißdorf $^{1}$. \\ Pablo Steinberg ${ }^{3}$ - Oliver J. Schmitz ${ }^{2} \cdot$ Thorsten Benter $^{1} \cdot$ Nils Helge Schebb $^{1}$
}

Received: 17 April 2020 / Revised: 15 June 2020 / Accepted: 29 June 2020 / Published online: 22 July 2020

(C) The Author(s) 2020

\begin{abstract}
Eicosanoids and other oxylipins play an important role in mediating inflammation as well as other biological processes. For the investigation of their biological role(s), comprehensive analytical methods are necessary, which are able to provide reliable identification and quantification of these compounds in biological matrices. Using charge-switch derivatization with AMPP $(N$-(4-aminomethylphenyl)pyridinium chloride) in combination with liquid chromatography ion mobility quadrupole time-offlight mass spectrometry (LC-IM-QTOF-MS), we developed a non-target approach to analyze oxylipins in plasma, serum, and cells. The developed workflow makes use of an ion mobility resolved fragmentation to pinpoint derivatized molecules based on the cleavage of AMPP, which yields two specific fragment ions. This allows a reliable identification of known and unknown eicosanoids and other oxylipins. We characterized the workflow using 52 different oxylipins and investigated their fragmentation patterns and ion mobilities. Limits of detection ranged between 0.2 and $10.0 \mathrm{nM}$ (1.0-50 pg on column), which is comparable with other state-of-the-art methods using LC triple quadrupole (QqQ) MS. Moreover, we applied this strategy to analyze oxylipins in different biologically relevant matrices, as cultured cells, human plasma, and serum.
\end{abstract}

Keywords Eicosanoids $\cdot$ Oxylipins $\cdot$ Lipidomics $\cdot$ Ion mobility-mass spectrometry $\cdot$ AMPP $\cdot$ DTIMS

\section{Introduction}

Oxylipins are oxidation products of polyunsaturated fatty acids (PUFA) derived from the arachidonic acid (ARA) cascade which can act as lipid mediators [1,2]. The analysis of

Stefan Hellhake and Sven W. Meckelmann contributed equally to this work.

Electronic supplementary material The online version of this article (https://doi.org/10.1007/s00216-020-02795-2) contains supplementary material, which is available to authorized users.

Nils Helge Schebb

nils@schebb-web.de

1 School of Mathematics and Natural Sciences, University of Wuppertal, Gauss-Str. 20, 42119 Wuppertal, Germany

2 Applied Analytical Chemistry \& Teaching and Research Center for Separation, University of Duisburg-Essen, Universitätsstr. 5-7, 45141 Essen, Germany

3 Institute for Food Toxicology, University of Veterinary Medicine Hannover, Bünteweg 2, 30559 Hannover, Germany these metabolites is important to understand the process of inflammation, pain, and related diseases [3, 4]. Numerous possible oxidation products of different PUFAs yield a wide range of oxylipins with different structure. This structural diversity, in addition to concentrations in the nanomolar range and below, is still challenging for modern analytical methods [5-7].

Three enzymatic pathways of the ARA cascade as well as non-enzymatic autoxidation lead to the formation of oxylipins $[8,9]$. Initially formed products can be further converted by a variety of specific downstream enzymes, for instance the soluble epoxide hydrolase (sEH) $[10,11]$. In the first enzymatic pathway, ARA or eicosapentaenoic acid (EPA) is converted by cyclooxygenases $(\mathrm{COX})$ to different prostaglandins and thromboxanes such as prostaglandin $\mathrm{D}_{2}\left(\mathrm{PGD}_{2}\right)$ or prostaglan$\operatorname{din} \mathrm{E}_{2}\left(\mathrm{PDE}_{2}\right)[1,2,12]$. The second path of the ARA cascade involves the oxidation of PUFAs by different lipoxygenases (LOX), leading via hydroperoxy PUFA intermediates to leukotrienes and lipoxins as well as hydroxyl PUFAs such as 15hydroxyeicosapentaenoic acid (15-HEPE) [13-15]. In the third branch of the ARA cascade, cytochrome P450 
monooxygenases (CYP) give rise to a diverse number of hydroxy and epoxy PUFAs, e.g. 19,20-epoxydocosapentaenoic acid (19(20)-EpDPE) and 20-hydroxyeicosatetraenoic acid (20-HETE) [16-18].

The different precursor PUFAs used in the ARA cascade and its three branches, along with downstream and autoxidation products, lead to a large variety of eicosanoids and other oxylipins having different biological roles. Nonetheless, most products exhibit similar chemical structures, which makes the analytical characterization challenging. Common methods for the analysis of such compounds utilize reversed-phase liquid chromatography in combination with electrospray ionization (ESI) and tandem mass spectrometry (MS/MS) on triple quadrupole (QqQ) instruments [19-23]. The combination of chromatography and mass spectrometry has proven to be suitable for the simultaneous, sensitive, and selective identification and quantification of different eicosanoid and oxylipin isomers in various biological samples [7, 24-28]. Still, the low concentrations as well as the large number of structurally similar molecules, including regioisomers, constitute a challenge for the separation and quantification of oxylipins $[5,7]$. In recent years, ion mobility quadrupole time-of-flight mass spectrometry (IM-QTOF-MS) has evolved as a new tool for non-targeted analysis [29-31]. Ion mobility is offering an additional separation dimension and enhances the separation of isomers. In addition, QTOF-MS provides tandem mass spectrometry along with accurate and highly resolved masses for the selective identification of compounds [32,33].

Studies on ion mobility-based mass spectrometry analyses of oxylipins often utilize ESI in the negative mode [30, 34-36]. Hinz et al. [27] developed an analytical LC-IMQTOF-MS method allowing the profiling and quantification of the oxylipin content in biological samples. Using ion mobility spectrometry, they were able to identify 57 features using an internal library of standards and quantify 16 analytes in thrombin-activated human platelets [30]. However, the application of ESI in the negative mode is in contradiction with the use of acidified eluents needed for the separation of oxylipin mixtures, thereby limiting the generation of negatively charged ions and ultimately reducing the sensitivity of the method. The formation of positive ions in common ESI sources is generally more effective than the formation of negative ions [37, 38]. Charge-switch derivatization is a tool, which allows using ESI in the positive mode and thereby overcoming the abovementioned limitations. Different derivatization reagents have been developed for this purpose, for example, $N$-(4-aminomethylphenyl)pyridinium chloride (AMPP) $[37,39,40]$.

We combine charge-switch derivatization using AMPP and ion mobility-mass spectrometry to develop a novel workflow for targeted and non-targeted oxylipin analysis. This combination is applied to analyze and quantify the oxylipin content in different biologically relevant matrices, including cultured cells as well as human plasma and serum. The quantification results are compared with a sensitive LC-ESI(-)-QqQ-MS method without derivatization, an approach which is typically used for oxylipin analysis [22, 41]. In addition, we use a stable isotope-labeled derivatization reagent $\left({ }^{2} \mathrm{H}_{5}\right.$-AMPP) to perform an approach analogous to ITRAQ (isobaric tags for relative and absolute quantitation). This approach provides an additional tool for a parallel sample analysis coupled to a relative oxylipin quantification that can be used for quantification and comparison in a non-targeted approach. Moreover, we demonstrate that the derivatization with AMPP and the specific fragmentation of the derivates is a powerful tool to identify oxylipins, eicosanoids, and carboxylic acids in complex biological matrices.

\section{Material and methods}

\section{Chemicals and biological materials}

LC-MS grade acetonitrile (ACN), methanol (MeOH), $n$-hexane, ethyl acetate, and methyl formate were purchased from Fisher Scientific (Schwerte, Germany). Dulbecco's modified Eagle's medium (DMEM), fetal bovine serum (FBS), and all other cell culture reagents were purchased from Biochrom/ Merck (Berlin, Germany). Oxylipin standards and internal standards were either purchased from Cayman Chemicals (local distributor: Biomol, Hamburg, Germany) or synthesized in-house as described in the Electronic Supplementary Material (ESM). Acetic acid (HAc) LC-MS grade, formic acid (FA) LC-MS grade, $N$-(3-dimethylaminopropyl)- $N^{\prime}$ ethylcarbodiimide hydrochloride (EDC), N,Ndimethylformamide (DMF), human blood serum, and all other chemicals were acquired from Sigma-Aldrich (Steinheim, Germany). Human blood plasma and second-pool human blood serum were purchased from BioIVT (West Sussex, UK). Caco-2 cells were obtained from the German Collection of Microorganisms and Cell Cultures (DSMZ, Braunschweig, Germany). Finally, AMPP and ${ }^{2} \mathrm{H}_{5}$-AMPP were synthesized as described before [42] (for details see ESM).

\section{Cell culture and sEH inhibition}

Caco- 2 cells were cultured in DMEM supplemented with $20 \%$ FBS, $100 \mathrm{IU} / \mathrm{mL}$ penicillin $/ 100 \mu \mathrm{g} / \mathrm{mL}$ streptomycin, $2 \mathrm{mM}$ L-glutamine, and $1 \%$ non-essential amino acids. Cell cultures were maintained at $37{ }^{\circ} \mathrm{C}$ and $5 \% \mathrm{CO}_{2}$ in a humidified incubator. For sEH inhibition, 80,000 cells per well were seeded in 6-well plates (TPP, Trasadingen, Switzerland) and incubated for 10 days, before the medium in each well $(2 \mathrm{~mL})$ was either supplemented with $0.05 \%$ DMSO (solvent control) or with 0.5 and $5 \mu \mathrm{M}$ 1-(1-propanoylpiperidin-4-yl)-3- 
4-(trifluoromethoxy)phenylurea (TPPU). After treatment with TPPU for $48 \mathrm{~h}$, the culture medium was removed and the cells were first washed with $1 \mathrm{~mL}$ PBS. Then, the cells were detached using trypsin/EDTA and harvested by centrifugation $(500 \times g$ for $5 \mathrm{~min})$. After the cell pellet was resuspended in $1 \mathrm{~mL}$ PBS, the cell number per well was determined and the remaining cells were centrifuged again $\left(15,000 \times g\right.$ at $4{ }^{\circ} \mathrm{C}$ for $10 \mathrm{~min})$. Finally, the resulting supernatant was discarded and the cell pellet was frozen and stored at $-80{ }^{\circ} \mathrm{C}$ until further analysis. The toxicity of TPPU towards Caco- 2 cells was investigated by means of the SRB assay, which was performed as previously described [43]. No toxic effects were observed at the concentrations used for inhibition (see ESM).

\section{Sample preparation and derivatization}

Oxylipins from plasma, serum, or cells were extracted by anion exchange solid-phase extraction in combination with hydrolysis as triplicates according to Rund et al. and Ostermann et al. [22, 44], albeit with some modifications. Briefly, $100 \mu \mathrm{L}$ serum or $1-5 \times 10^{5}$ cells were mixed with $400 \mu \mathrm{L}$ isopropanol for protein precipitation as well as $150 \mu \mathrm{L}$ internal standards $\left({ }^{2} \mathrm{H}_{11}-11,12\right.$-DiHETrE and ${ }^{2} \mathrm{H}_{4}-9$-HODE; each $\left.150 \mathrm{nM}\right)$ and kept at $-80^{\circ} \mathrm{C}$ for $30 \mathrm{~min}$. For further studies, we recommend the use of more internal standards as described in $[22,23,27$, 28]. After centrifugation $\left(15,000 \times \mathrm{g}\right.$ at $4{ }^{\circ} \mathrm{C}$ for $\left.10 \mathrm{~min}\right)$, $100 \mu \mathrm{L} \mathrm{KOH} \mathrm{(1.5} \mathrm{M} \mathrm{in} \mathrm{MeOH/water;} \mathrm{75/25;} \mathrm{v/v)} \mathrm{was} \mathrm{added}$ to the supernatant to hydrolyze esterified oxylipins. The solution was then incubated at $60^{\circ} \mathrm{C}$ for $60 \mathrm{~min}$ and subsequently acidified with $40 \mu \mathrm{L} 50 \% \mathrm{HAc}$ to a $\mathrm{pH}>6$. Next, the sample was loaded onto a preconditioned ( $2 \mathrm{~mL}, 0.1 \mathrm{M} \mathrm{Na}_{2} \mathrm{HPO}_{4} /$ $\mathrm{HAc}, \mathrm{pH}=6$ ) anion exchange Bond Elut Certify II SPE cartridge (200 mg, $3 \mathrm{~mL}$, Agilent, Waldbronn, Germany). After loading, the sample was washed with $2 \mathrm{~mL}$ water and $2 \mathrm{~mL}$ water $/ \mathrm{MeOH}(50 / 50, \mathrm{v} / \mathrm{v})$ and dried before elution with $2 \mathrm{~mL}$ ethyl acetate/ $n$-hexane $(75 / 25 ; \mathrm{v} / \mathrm{v})$. The organic phase was collected in a glass test tube and spiked with $6 \mu \mathrm{L}$ of $30 \%$ glycerol in $\mathrm{MeOH}$. Finally, the sample was dried using a vacuum centrifuge for $60 \mathrm{~min}$ and subsequently derivatized.

For derivatization, the dried SPE extract or the standard were dissolved in $25 \mu \mathrm{L} \mathrm{ACN/DMF} \mathrm{(5/1;} \mathrm{v/v)} \mathrm{and} 25 \mu \mathrm{L}$ EDC $640 \mathrm{mM}$ in water. The solution was transferred into a vial and $25 \mu \mathrm{L} 45 \mathrm{mM}$ AMPP or ${ }^{2} \mathrm{H}_{5}$-AMPP and $25 \mu \mathrm{L}$ $20 \mathrm{mM}$ 1-hydroxybenzotriazole were added before incubation at $40{ }^{\circ} \mathrm{C}$ for $40 \mathrm{~min}$ [42]. The derivatized sample was then either directly analyzed or stored at $-80^{\circ} \mathrm{C}$ until analysis.

\section{LC-MS analysis}

For LC-IM-QTOF-MS(/MS) analysis, the liquid chromatography was carried out with an Agilent 1290 Infinity LC system (Agilent Technologies Inc., Waldbronn, Germany) equipped with a Kinetex C8 column $(2.6 \mu \mathrm{m}, 100 \times 2.1 \mathrm{~mm}, 100 \AA$,
Phenomenex, Aschaffenburg, Germany). Separation was achieved by utilizing a binary gradient at a flow rate of $300 \mu \mathrm{L} / \mathrm{min}$ with $\mathrm{ACN} /$ water/FA $(5 / 95 / 0.1 ; \mathrm{v} / \mathrm{v} / \mathrm{v})$ as solvent $\mathrm{A}$ and $\mathrm{ACN} /$ water/FA $(95 / 5 / 0.1 ; \mathrm{v} / \mathrm{v} / \mathrm{v})$ as solvent $\mathrm{B}$. The gradient was $0-1 \mathrm{~min}$ isocratic $15 \% \mathrm{~B}, 1-2$ min linear from 15 to $32 \% \mathrm{~B}, 6-21$ min linear from 32 to $40 \% \mathrm{~B}, 21-22$ min linear from 40 to $100 \% \mathrm{~B}, 22-26 \mathrm{~min}$ isocratic $100 \% \mathrm{~B}$, followed by reconditioning at $15 \% \mathrm{~B}$ for $9 \mathrm{~min}$. The column temperature was kept at $50{ }^{\circ} \mathrm{C}$. Carry over for all analytes was measured with organic solvent blanks after each calibration standard of highest concentration and each sixth biological sample. In all cases, no carry over was observed.

Mass spectrometric detection was carried out with an Agilent 6560 IM-QTOF-MS (in positive tuning the mass resolution at $\mathrm{m} / \mathrm{z}, 322.0481$ is $\sim 17,000$ and at $\mathrm{m} / \mathrm{z}, 922.0097$ is 25,516 ) equipped with the Agilent Dual Jet Stream Source for ionization in the positive mode using the following parameters: $\mathrm{V}_{\text {cap }} 2500 \mathrm{~V}$, nozzle voltage $1250 \mathrm{~V}$, gas temp. $325^{\circ} \mathrm{C}$, drying gas flow $12 \mathrm{~L} / \mathrm{min}$, nebulizer gas pressure $12 \mathrm{psi}$, sheath gas temp. $275{ }^{\circ} \mathrm{C}$, and sheath gas flow $9 \mathrm{~L} / \mathrm{min}$. Nitrogen was used as IM drift gas. Detection was carried out using the alternating frame detection mode, switching between high and zero fragmentation energy at 2.1 frames/s. The collision energy for high fragmentation was linearly ramped from $10 \mathrm{~V}$ at $10 \mathrm{~ms}$ drift time to $59 \mathrm{~V}$ at $50 \mathrm{~ms}$ drift time.

Targeted LC-QqQ-MS analysis was carried out on an Agilent 1290 Infinity LC system coupled to a Sciex 6500 QTRAP instrument (Sciex, Darmstadt, Germany) as previously described $[22,41]$.

\section{Data analysis}

Data evaluation for known oxylipins was carried out using the Skyline-Daily version 4.1.1.18179 (MacCoss Lab Software, University of Washington, Seattle, Washington) [45-47]. The feature analysis for the non-targeted analysis was done with the Agilent Mass Profiler version 8.1.153.0 using the following parameters: retention time $\left(t_{R}\right)$ window $2.0-25.0 \mathrm{~min}$, charge state +1 , retention time tolerance $\pm 0.3 \mathrm{~min}$, drift time tolerance $1.5 \%$, mass tolerance $10 \mathrm{ppm}, q$-score $\geq 40$, and intensity $\geq 100$ counts. Resulting features were exported individually for each sample as the software does not allow merging of the MS/MS information into one file. Further processing was done using the Python programming language (Python Software Foundation, https://www.python.org/, see ESM) to filter features in each sample for the characteristic AMPP fragment ions at $\mathrm{m} / \mathrm{z} 169.0$ and 183.1 with a mass accuracy of $0.1 \mathrm{Da}$. The Python script then compared the individual samples and searched for shared $\left[\mathrm{M}^{+}\right]$with $>80 \%$ of their 25 most abundant fragments in the high fragmentation data with an accuracy of $0.1 \mathrm{Da}$. These shared $\left[\mathrm{M}^{+}\right]$also needed to have the same CCS with an accuracy of $2.5 \AA^{2}$ 
(equivalent to $<1 \%$ error) and the same retention time with an accuracy of $0.2 \mathrm{~min}$. The found features were then exported into an external file.

Statistical analysis was carried out using GraphPad PRISM version 5.00 (GraphPad Software, San Diego, California).

\section{Results}

\section{Fragmentation of AMPP-derivatized oxylipins}

The derivatization of the hydroxy, dihydroxy, and epoxy PUFA standards with AMPP and ${ }^{2} \mathrm{H}_{5}$-AMPP and the LCMS/MS analysis was performed to determine the fragmentation pattern of derivatized oxylipins and to identify characteristic fragments for identification. Table 1 shows the specific fragments for each investigated compound and Fig. 1 depicts an exemplary fragment spectrum of 14(15)-EpETrE-AMPP and $14(15)$-EpETrE- ${ }^{2} \mathrm{H}_{5}$-AMPP compared with the nonderivatized 14(15)-EpETrE. In both spectra of the AMPPand ${ }^{2} \mathrm{H}_{5}$-AMPP-derivatized oxylipins, the molecular $\left[\mathrm{M}^{+}\right]$ ion was the most abundant. In addition, characteristic fragments of the AMPP cleavage at $\mathrm{m} / \mathrm{z} 183$ and $\mathrm{m} / \mathrm{z}, 169$ as well as at $m / z, 188$ and $m / z, 174$ for ${ }^{2} \mathrm{H}_{5}$-AMPP cleavage were observed. Other ions commonly generated by ESI such as adducts of $\mathrm{H}^{+}, \mathrm{Na}^{+}$, and $\mathrm{K}^{+}$or neutral loss of water for the hydroxy PUFAs could not be observed with significant abundance.

\section{Separation of AMPP-derivatized oxylipins}

Reversed-phase liquid chromatography is necessary for the characterization of oxylipins in complex biological matrices. We used a separation method under common conditions with a C8 column and a binary gradient [19, 21, 48, 49]. Under these conditions, sufficient separation of 52 derivatized hydroxy, dihydroxy, and epoxy PUFAs in 22 min was achieved (Fig. 2). Full widths at half maximum (FWHM) ranged from $0.03 \mathrm{~min}$ for the peak of 11-HETE to $0.37 \mathrm{~min}$ for the peak of 17(18)-EpETE. In addition, co-eluting analytes were separated by ion mobility or mass spectrometry (Table 1), e.g. 8,9$\operatorname{EpETrE~}\left(m / z 487.3325\right.$ for the $\left[\mathrm{M}^{+}\right], t_{\mathrm{R}} 18.28 \mathrm{~min}, t_{\mathrm{d}}$ $27.18 \mathrm{~ms})$ and 10,11-EpDPE $\left(\mathrm{m} / z, 511.3325\right.$ for the $\left[\mathrm{M}^{+}\right], t_{\mathrm{R}}$ $\left.18.34 \mathrm{~min}, t_{\mathrm{d}} 27.79 \mathrm{~ms}\right)$. Only 8-HEPE and 12-HEPE $\left(t_{\mathrm{R}}\right.$ $11.25 \mathrm{~min}$ ) remained as a critical compound pair, which could not be separated neither by chromatography nor by ion mobility, mass spectrometry, or tandem mass spectrometry.

\section{Evaluation of the drift behavior of AMPP-derivatized oxylipins}

Fifty-two different hydroxy, dihydroxy, and epoxy PUFAs were derivatized with AMPP and analyzed using IM-QTOF-
MS(/MS) to characterize the behavior of derivatized oxylipins during IM separation. Drift times of all analytes were determined and the corresponding collision cross sections were calculated (CCS; Table 1). The IM separation of co-eluting compounds also allowed the detection of clean MS/MS spectra in the all-ion fragmentation mode when using the alternating frame method (Fig. 3).

The CCS values of the analytes were evaluated according to the precursor PUFA (Fig. 4a) as well as to the position of the functional group of the oxylipins (Fig. 4b-d). The CCS increased with the length of the PUFA (Fig. 4). The CCS values of derivatized oxylipins of EPA were in the range between 207.7 and $219.7 \AA^{2}$, while the docosahexaenoic acid (DHA) products showed larger CCS values ranging from 218.6 to $226.0 \AA^{2}$. The CCS values of the ARA products ranged between 213.8 and $223.1 \AA^{2}$ and $\alpha$-linolenic acid (ALA) products showed CCS values of 209.4 to $216.4 \AA^{2}$. Regarding the position of the functional group from the carboxyl group to the end of the carbon chain in a specific group of regioisomers (e.g. HETEs), the CCS showed a maximum at C10-C12 (Fig. 4b). The same trend was observed for different PUFAs as well as for dihydroxy or epoxy-bearing PUFAs.

\section{Quantification of oxylipins}

Calibration curves for all 52 different hydroxy, dihydroxy, and epoxy PUFAs after derivatization were prepared to determine analytical limits. Thereafter, the concentration of different oxylipins in plasma, serum, and Caco-2 cell pellets was quantified, in order to demonstrate the capabilities of the approach and to compare the results with the analysis of non-derivatized samples analyzed by LC-QqQ-MS (Figs. 5 and 6). To further demonstrate that specific enzymatic processes can be monitored, the analyzed Caco-2 cells were treated with different amounts of TPPU, an inhibitor of the soluble epoxide hydrolase.

Limits of detection (LOD, $\mathrm{s} / n>3$ ) ranged between $0.2 \mathrm{nM}$ for 12,13-DiHODE as well as 5,6-DiHETE and 10.0 nM for 5-HETE (corresponding to 1.0-50 pg on column). Lower limits of quantification (LLOQ, s/n>9) ranged between $0.3 \mathrm{nM}$ for 5,6-DiHETE and $63 \mathrm{nM}$ for 5(6)-EpETrE (corresponding to $1.0-315 \mathrm{pg}$ on column). Linearity of the calibration was $>0.98\left(R^{2}\right)$ for all analytes with an accuracy $>0.75$ for all data points (Table 1).

The results of the quantitative analysis are shown in Fig. 6. Generally, the values for hydroxyl PUFAs obtained by LCIM-QTOF-MS(/MS) were in the same range as concentrations determined by the reference method (LC-QqQ-MS), with some exceptions: lower concentrations were consistently found for 9-HODE and 13-HODE across all samples using the developed LC-IM-QTOF-MS(/MS) method. The analysis of derivatized oxylipins using the LC-IM-QTOF-MS(/MS) method showed higher concentrations of dihydroxy and 
Table 1 LC-IM-QTOF-MS(/MS) parameters for 52 oxylipin standards after charge-switch derivatization using AMPP. Shown are the transitions for $\left[\mathrm{M}^{+}\right]$and fragment ion, retention time $\left(t_{\mathrm{R}}\right)$, drift time $\left(t_{\mathrm{D}}\right)$, collision cross section $(\Omega)$, limit of detection (LOD), lower limit of quantification (LLOQ), upper limit of quantification (ULOQ), and $R^{2}$ for the resulting calibration. Ions used for quantification are shown in italicized numbers

\begin{tabular}{|c|c|c|c|c|c|c|c|c|c|}
\hline Analyte & {$\left[\mathrm{M}^{+}\right]$} & Fragment ion & $t_{\mathrm{R}}(\min )$ & $t_{\mathrm{D}}(\mathrm{ms})$ & $\Omega\left(\AA^{2}\right)$ & LOD (nM) & LLOQ (nM) & ULOQ (nM) & $R^{2}$ \\
\hline 9,10-DiHOME & 481.3430 & 369.2178 & 9.06 & 27.11 & 216.8 & 0.6 & 1.5 & 292 & 0.9867 \\
\hline 12,13-DiHOME & 481.3430 & 351.2436 & 8.63 & 27.18 & 217.4 & 0.8 & 2.0 & 402 & 0.9957 \\
\hline 9,10-DiHODE & 479.3273 & 369.2178 & 7.74 & 27.06 & 216.4 & 0.5 & 0.5 & $1256^{*}$ & 0.9987 \\
\hline 12,13-DiHODE & 479.3273 & 351.2436 & 7.40 & 26.38 & 211.0 & 0.2 & 0.9 & $434 *$ & 0.9816 \\
\hline 15,16-DiHODE & 479.3273 & 391.2749 & 7.49 & 26.20 & 209.6 & 0.6 & 1.4 & $1435^{*}$ & 0.9895 \\
\hline 5,6-DiHETrE & 505.3430 & 313.1552 & 12.34 & 27.50 & 219.6 & 4.7 & 12 & $1183 *$ & 0.9958 \\
\hline 8,9-DiHETrE & 505.3430 & 295.1810 & 11.14 & 27.79 & 221.9 & 0.9 & 2.3 & 467 & 0.9873 \\
\hline 11,12-DiHETrE & 505.3430 & 335.2123 & 10.28 & 27.94 & 223.1 & 1.5 & 3.7 & 749 & 0.9966 \\
\hline 14,15-DiHETrE & 505.3430 & 375.2436 & 9.48 & 27.57 & 220.2 & 2.4 & 5.9 & $1176^{*}$ & 0.9950 \\
\hline 5,6-DiHETE & 503.3274 & 313.1552 & 10.02 & 27.08 & 216.3 & 0.2 & 0.3 & $16^{*}$ & 0.9743 \\
\hline 8,9-DiHETE & 503.3274 & 295.1810 & 9.17 & 27.41 & 218.9 & 4.0 & 7.9 & 396 & 0.9974 \\
\hline 11,12-DiHETE & 503.3274 & 335.2123 & 8.81 & 27.54 & 220.0 & 1.0 & 9.9 & 497 & 0.9780 \\
\hline 14,15-DiHETE & 503.3274 & 375.2436 & 8.36 & 27.27 & 217.8 & 7.0 & 14 & 701 & 0.9990 \\
\hline 17,18-DiHETE & 503.3274 & 415.2749 & 7.98 & 26.66 & 212.9 & 1.8 & 4.6 & 914 & 0.9930 \\
\hline 10,11-DiHDPE & 529.3430 & 321.1967 & 11.13 & 28.33 & 226.0 & 1.2 & 6.2 & $3083^{*}$ & 0.9986 \\
\hline 13,14-DiHDPE & 529.3430 & 361.2280 & 10.63 & 28.19 & 224.9 & 0.9 & 4.3 & $2161 *$ & 0.9959 \\
\hline 16,17-DiHDPE & 529.3430 & 401.2593 & 10.23 & 28.05 & 223.7 & 0.7 & 3.4 & $1710^{*}$ & 0.9963 \\
\hline 19,20-DiHDPE & 529.3430 & 441.2906 & 9.62 & 27.60 & 220.2 & $7.1 *$ & 7.1 & $1419 *$ & 0.9956 \\
\hline 9(10)-ЕpOME & 463.3325 & 339.2073 & 15.89 & 26.71 & 213.8 & 0.8 & 3.8 & 376 & 0.9935 \\
\hline 12(13)-EpOME & 463.3325 & 363.2436 & 15.38 & 26.66 & 213.4 & 0.7 & 3.6 & 359 & 0.9949 \\
\hline 9(10)-EpODE & 461.3168 & 339.2073 & 13.19 & 26.41 & 211.5 & 1.1 & 5.4 & 535 & 0.9984 \\
\hline 12(13)-EpODE & 461.3168 & 363.2436 & 12.79 & 26.24 & 210.1 & 0.5 & 1.2 & 243 & 0.9937 \\
\hline 15(16)-EpODE & 461.3168 & 389.2593 & 12.39 & 26.15 & 209.4 & 1.5 & 3.7 & 740 & 0.9958 \\
\hline 5(6)-EpETrE & 487.3325 & 283.1447 & 18.95 & 27.18 & 217.3 & 6.3 & 63 & $626^{*}$ & 0.9324 \\
\hline 8(9)-EpETrE & 487.3325 & 307.1810 & 18.27 & 27.18 & 217.3 & 3.6 & 14 & $357^{*}$ & 0.9991 \\
\hline 11(12)EpETrE & 487.3325 & 347.2123 & 17.79 & 27.24 & 217.8 & 6.2 & 25 & $615^{*}$ & 0.9992 \\
\hline 14(15)-EpETrE & 487.3325 & 333.1967 & 16.35 & 26.98 & 215.7 & 0.4 & 2.0 & 195 & 0.9952 \\
\hline 8(9)-ЕpETE & 485.3168 & 307.1810 & 14.85 & 26.81 & 214.4 & 1.7 & 4.2 & 833 & 0.9874 \\
\hline 11(12)-EpETE & 485.3168 & 347.2123 & 14.51 & 26.66 & 213.2 & 2.2 & 5.5 & 552 & 0.9934 \\
\hline 14(15)-EpETE & 485.3168 & 387.2436 & 14.20 & 26.75 & 213.9 & $2.7 *$ & $2.7 *$ & 1347 & 0.9594 \\
\hline 17(18)-EpETE & 485.3168 & 413.2593 & 13.17 & 26.65 & 213.1 & $3.9^{*}$ & $3.9^{*}$ & 968 & 0.9988 \\
\hline 10(11)-EpDPE & 511.3325 & 363.2073 & 18.45 & 27.79 & 221.9 & 1.8 & 4.6 & 919 & 0.9717 \\
\hline 13(14)-EpDPE & 511.3325 & 402.2307 & 18.16 & 27.76 & 221.6 & 1.2 & 3.0 & 302 & 0.9792 \\
\hline 16(17)-EpDPE & 511.3325 & 413.2593 & 17.85 & 27.68 & 221.0 & 1.3 & 3.3 & 662 & 0.9985 \\
\hline 19(20)-EpDPE & 511.3325 & 399.2436 & 16.65 & 27.61 & 220.4 & 2.8 & 7.0 & 1119 & 0.9874 \\
\hline 9-HODE & 463.3325 & 339.2064 & 13.33 & 26.80 & 214.5 & $1.0^{*}$ & $1.0^{*}$ & 100 & 0.9730 \\
\hline 13-HODE & 463.3325 & 363.2449 & 12.95 & 26.81 & 214.6 & $1.0^{*}$ & $1.0^{*}$ & 100 & 0.9574 \\
\hline 5-HETE & 487.3325 & 283.1596 & 17.06 & 27.07 & 216.4 & 10.0 & 20 & $500^{*}$ & 0.9969 \\
\hline 8-HETE & 487.3325 & 295.1765 & 15.61 & 27.24 & 217.8 & 2.0 & 10 & $500^{*}$ & 0.9438 \\
\hline 9-HETE & 487.3325 & 335.2133 & 14.99 & 27.37 & 218.8 & 2.0 & 10 & $500^{*}$ & 0.9839 \\
\hline 11-HETE & 487.3325 & 345.1758 & 15.95 & 27.28 & 218.1 & 1.0 & 2.0 & $500^{*}$ & 0.9304 \\
\hline 12-HETE & 487.3325 & 347.2091 & 15.33 & 27.50 & 219.8 & 2.0 & 10 & $500^{*}$ & 0.9829 \\
\hline 15-HETE & 487.3325 & 363.3346 & 14.03 & 27.14 & 217.0 & 2.0 & 10 & $500^{*}$ & 0.9751 \\
\hline 20-HETE & 487.3325 & 333.1954 & 11.93 & 26.74 & 213.8 & 2.0 & 10 & $500 *$ & 0.9881 \\
\hline 5-HEPE & 485.3168 & 283.1582 & 13.82 & 26.45 & 211.5 & 2.0 & 10 & $500 *$ & 0.9979 \\
\hline 8-HEPE & 485.3168 & 295.1815 & 12.48 & 26.82 & 214.4 & 1.0 & 2.0 & $500^{*}$ & 0.8995 \\
\hline 12-HEPE & 485.3168 & 375.2058 & 12.48 & 27.15 & 217.1 & 1.0 & 2.0 & $500 *$ & 0.9961 \\
\hline
\end{tabular}


Table 1 (continued)

\begin{tabular}{rllllllllr}
\hline Analyte & {$\left[\mathrm{M}^{+}\right]$} & Fragment ion & $t_{\mathrm{R}}(\mathrm{min})$ & $t_{\mathrm{D}}(\mathrm{ms})$ & $\Omega\left(\AA^{2}\right)$ & LOD $(\mathrm{nM})$ & LLOQ (nM) & ULOQ (nM) & $R^{2}$ \\
\hline 15-HEPE & 485.3168 & 333.1957 & 11.90 & 26.74 & 213.8 & 1.0 & 2.0 & $500^{*}$ & 0.9805 \\
18-HEPE & 485.3168 & 439.3329 & 10.95 & 25.98 & 207.7 & $1.0^{*}$ & $1.0^{*}$ & $500^{*}$ & 0.9908 \\
7-HDHA & 511.3325 & 280.1516 & 16.72 & 27.38 & 218.6 & 1.0 & 2.0 & $500^{*}$ & 0.9632 \\
11-HDHA & 511.3325 & 361.1898 & 16.21 & 27.91 & 222.8 & $1.0^{*}$ & $1.0^{*}$ & $500^{*}$ & 0.9985 \\
14-HDHA & 511.3325 & 401.2270 & 15.67 & 27.83 & 222.2 & 2.0 & 10 & $500^{*}$ & 0.9842 \\
\hline
\end{tabular}

*Lowest/highest point of calibration

epoxy PUFAs in both plasma and serum. For example, $28 \pm$ $4 \mathrm{nM}$ 14,15-DiHETE was determined in human plasma using the LC-IM-QTOF-MS(/MS), whereas the LC-QqQ-MS analysis resulted in concentrations below the LLOQ. Similar results were obtained for 9(10)-EpODE: $315 \pm 60 \mathrm{nM}$ were detected using the IM-QTOF-MS(/MS) and 6.2 $\pm 0.6 \mathrm{nM}$ using the LC-QqQ-MS method.

Figure 5 shows the quantification results of the epoxides 5,6-, 8,9-, 11,12-, and 14,15-EpETrE in Caco-2 cells. After treatment with $5 \mu \mathrm{M}$ TPPU, significantly higher concentrations of 5,6-, 8,9- and 11,12-EpETrE were found. The concentration of 14,15-EpETrE showed the same trend, with an increase of up to $176 \pm 90 \mathrm{pmol} / 10^{6}$ cells and $427 \pm 222 \mathrm{pmol} /$ $10^{6}$ cells for 0.5 or $5 \mu \mathrm{M}$ TPPU, respectively.

\section{Relative quantification of oxylipins}

For the relative quantification of oxylipins in analogy to the iTRAQ approach, control Caco-2 samples were derivatized with AMPP. Additionally, a sample of Caco-2 cells incubated with $5 \mu \mathrm{M}$ TPPU was derivatized with ${ }^{2} \mathrm{H}_{5}$-AMPP. Both samples were mixed together and analyzed by LC-IM-QTOF$\mathrm{MS}(\mathrm{MS})$. For correction of different derivatization yields and detection responses, ${ }^{2} \mathrm{H}_{4}-9$-HODE was used as internal standard. An exemplary comparison of parallel and individual analyses of HEPEs is shown in Fig. 7. Considering the method error, both methods showed similar ratios between the controls and $5 \mu \mathrm{M}$ TPPU-treated Caco- 2 cells. For example, the ratio of 8 -HEPE was $4.1 \pm 0.7$ for the parallel analysis and 2.1 \pm 1.5 for the individual analysis.

\section{Non-targeted analysis of AMPP-derivatized oxylipins}

Human plasma samples were analyzed in triplicate, and the resulting data were further processed by feature analysis using the Agilent Mass Profiler software. The software extracted molecular features $(\mathrm{m} / \mathrm{z}$, retention time, drift time, and abundance) from the data along with the corresponding MS/MS spectra. Using a custom Python script, we combined the information to identify features with AMPP-specific fragments. This approach yields additional insights by using derivatization in combination with LC-IM-QTOF-MS(/MS) analysis in non-targeted approaches compared with LC-QqQ-MS analyses.

In total, 4137 features were found in the plasma samples. Filtering for the specific AMPP cleavage $(\mathrm{m} / \mathrm{z} 169.0$ and $\mathrm{m} / \mathrm{z}$
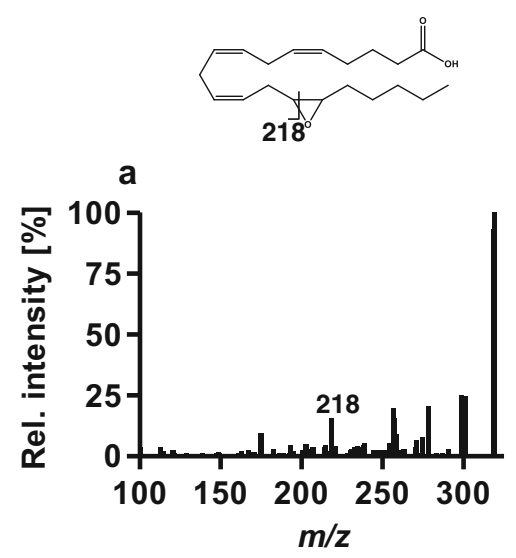

Fig. 1 ESI(-) collision-induced dissociation (CID) fragment spectrum of 14(15)-EpETrE analyzed using a Sciex 6500 QqQ instrument (a) in comparison with the ESI(+) collision-induced dissociation (CID) 14(15)-

b
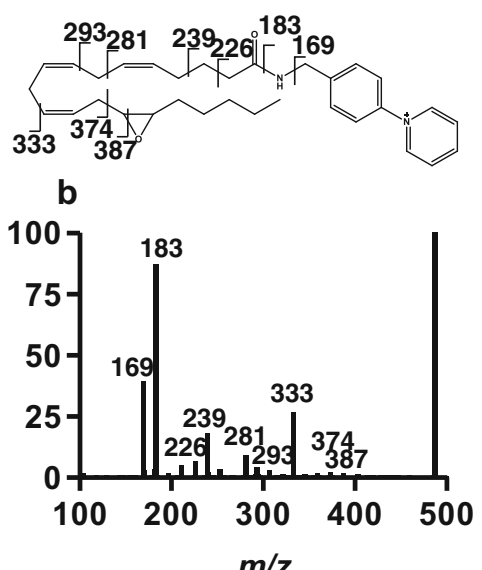

$m / z$

EpETrE spectrum measured using an Agilent 6560 IM-QTOF-MS instrument and after derivatization with AMPP (b) and ${ }^{2} \mathrm{H}_{5}$-AMPP (c) 
Fig. 2 Chromatographic separation of regioisomeric hydroxy, dihydroxy, and epoxy PUFAs after derivatization with AMPP. Shown are the extracted ion chromatograms filtered by drift time and $\mathrm{m} / \mathrm{z}$, ratio for the most selective ion species as shown in Table 1. Separation was carried out on a C8 Kinetex column $(2.6 \mu \mathrm{m}, 100 \times 2.1 \mathrm{~mm}$, $100 \AA$ ) with acidified water/ACN as mobile phase
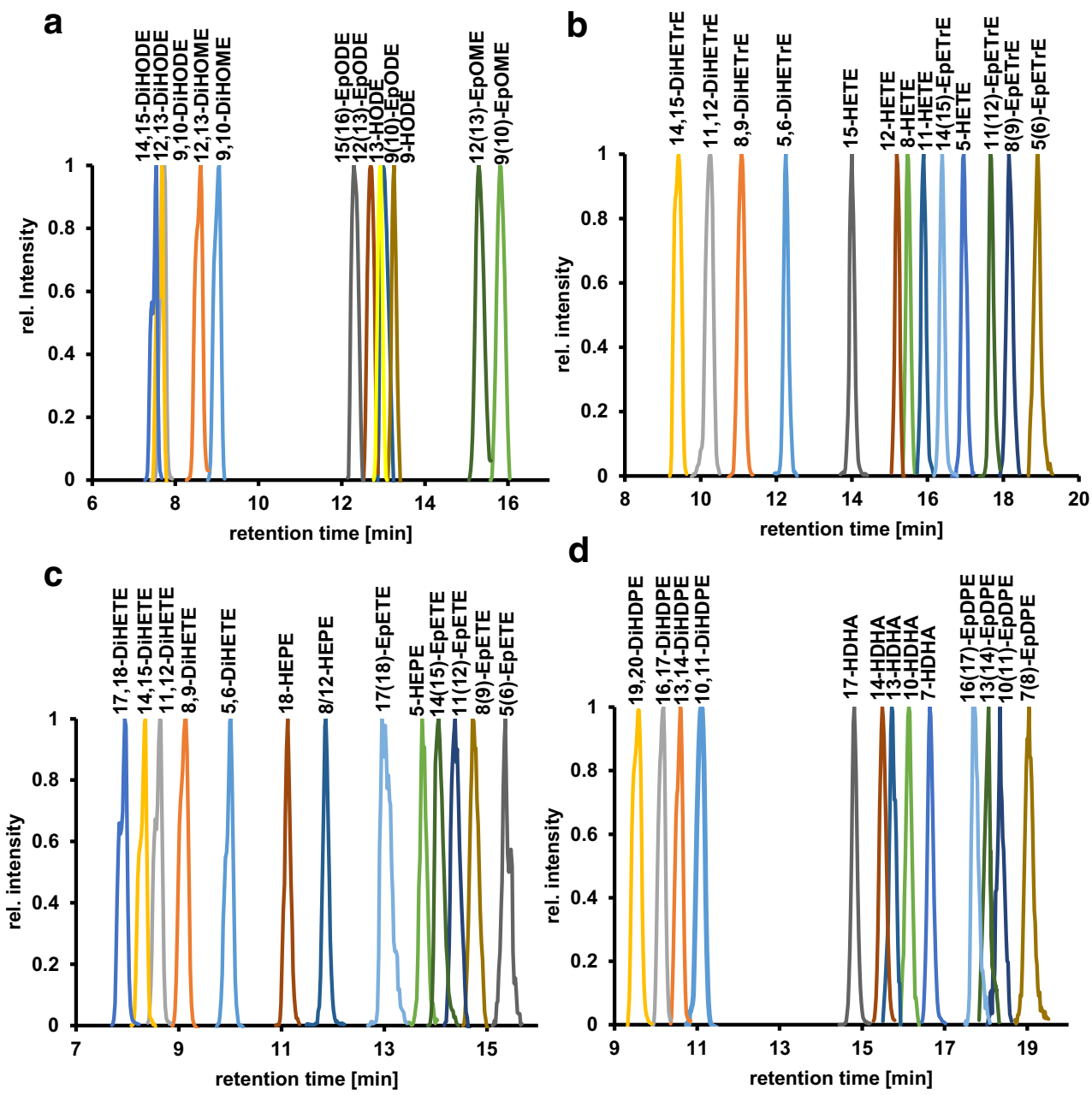

$183.1)$ reduced the number to $\sim 1200$. In the next step, the fragmentation data were compared between all three samples. A bioinformatics workflow (i.e., a further custom Python script) was used to find shared features, including fragmentation data. The script filtered for features, which had $80 \%$ or more of their 25 most abundant fragment ions in common. In this way, 285 shared features remained. A list of features found in plasma with sufficient precision is provided in ESM (Table S1). This workflow allows the identification of other oxylipins in the samples with the capability to elucidate the structure. An application example is shown in Table 2 and ESM Fig. S2 for 10-hydroxy-8-octadecenoic acid.

\section{Discussion}

The aim of the present study was the development of an LCIM-QTOF-MS(/MS) method for the analysis of oxylipins after charge-switch derivatization using AMPP in biological samples. The method was combined with computer-aided feature analysis tools for the non-targeted analysis of oxylipins.

\section{Characterization of the derivatization}

Fragmentation of analytes derivatized with AMPP, compared with non-derivatized analytes, resulted in more fragments, and thus, in a higher level of information for structural analysis than seen in the case of other permanent charge derivatizations $[50,51]$. Characteristic and often abundant peaks in the fragmentation spectra are ions derived from the cleavage of the AMPP head group ( $m / z, 183.0$ and $m / z$ 169.0; Fig. 1). The same fragmentation behavior was observed for ${ }^{2} \mathrm{H}_{5}$-AMPPderivatized analytes, yielding series of fragment ion signals with a spacing of 5 Da because of the heavier deuterium isotopes (Fig. 1). Specific fragments of hydroxy and dihydroxy PUFAs were the same compared with those obtained with ESI(-) methods with the expected shift caused by the addition of AMPP. For example, after derivatization with AMPP, 5HETE resulted in ions at $\mathrm{m} / z, 487.3$ for the $\left[\mathrm{M}^{+}\right]$ion and 295.2 for a specific fragment ion, in comparison with $\mathrm{m} / \mathrm{z}$ 319.2 and $\mathrm{m} / \mathrm{z} 115.1$ in the ESI(-) mode. Both fragment ions are products from an alpha cleavage next to the hydroxyl group [19, 20, 22]. For epoxy PUFAs, the fragmentation behavior of derivatized analytes is different when compared with 


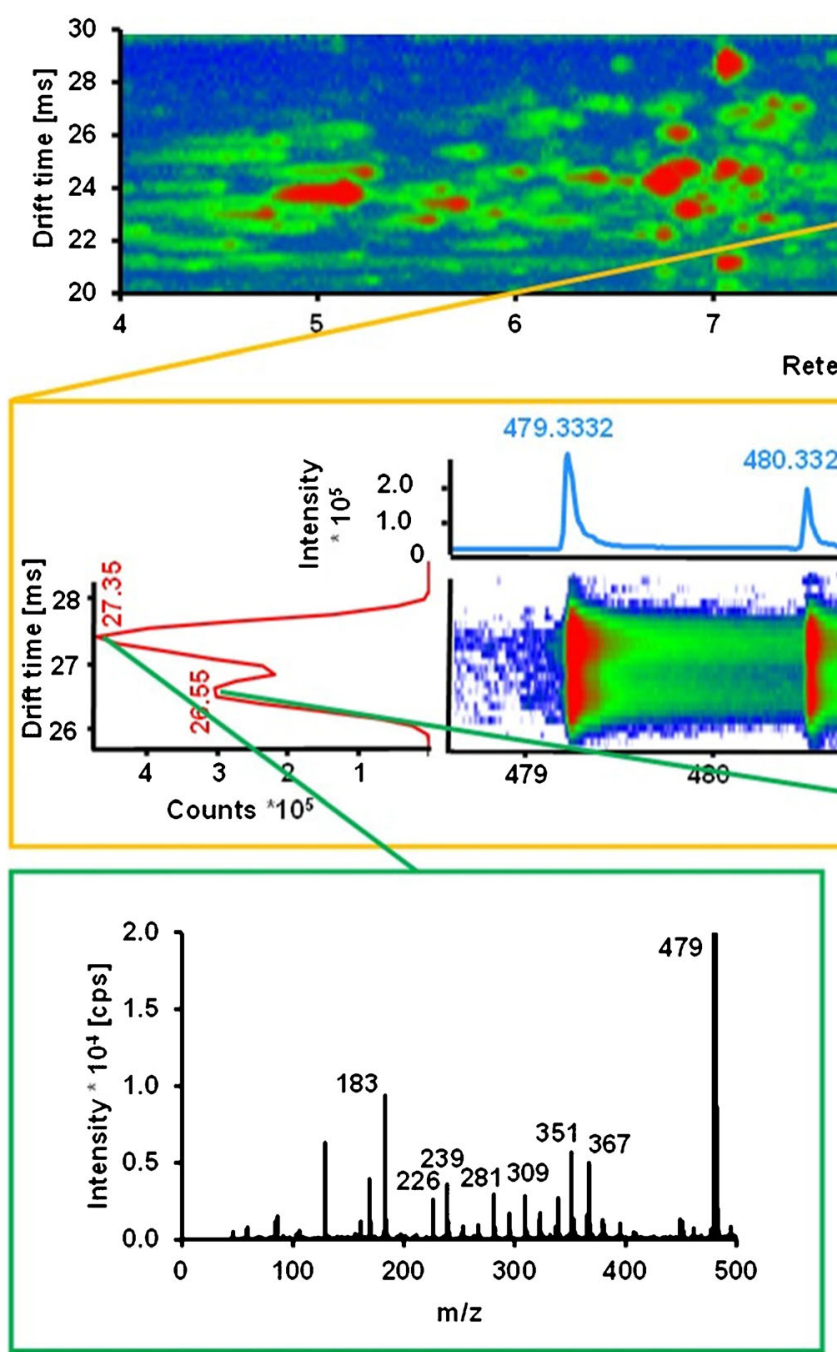

Fig. 3 Separation of co-eluting and isobaric analytes $(m / z$ 479.3332) in human plasma by IM-QTOF-MS/MS. Top: chromatographic separation as 2D heat map with two analytes co-eluting at $9.6 \mathrm{~min}$. Middle: drift time spectrum of the analytes co-eluting at $9.6 \mathrm{~min}$. The drift times of 26.55

analyses performed with ESI(-) methods. The derivatization led to new specific and sensitive transitions for the epoxy PUFA, e.g. $\mathrm{m} / z 487.3$ and $m / z 333.2$ as $\left[\mathrm{M}^{+}\right]$and the fragment ion of 14(15)-EpETrE, respectively, instead of $m / z 319.2$ and 219.2 in the $\operatorname{ESI}(-)$ mode $[19,20,22]$.

\section{LC-IM-QTOF-MS(/MS) separation and detection}

The liquid chromatographic separation of the derivatized oxylipins was performed under common conditions using a reversed-phase column and an acidified eluent of water and ACN. Generally, the derivatization of analytes leads to structurally more similar molecules. Consequently, the separation of oxylipins becomes increasingly challenging after derivatization with AMPP [52]. Moreover, electrostatic interactions of the permanent positive charge of the AMPP head group with the column material (i.e., the remaining acidic silanol groups) hamper the separation [53]. However, the applied chromatographic method still provides a sufficient extent of separation of the 52 investigated oxylipins after derivatization in $22 \mathrm{~min}$. The use of the no fragmentation mode of the LCIM-QTOF-MS(/MS) alone is insufficient, as many oxylipin molecular ion masses are isobaric. For example, all EpETrE and HETEs have the same molecular formula and, thus, an accurate mass of 487.3325 Da. Therefore, an additional separation as well as MS/MS is necessary to detect and identify individual oxylipins. We used LC-IM-QTOF-MS(/MS) in the alternating frame mode to separate the precursor ions of the analytes by ion mobility. In one frame of this mode, the precursor ions are directly transferred to the TOF analyzer (no fragmentation/full scan mode), while in the subsequent frame, the collision cell is activated and fragmentation is induced. Because the fragmentation takes place downstream of the drift tube, fragment ions and precursor ions have identical drift 
Fig. 4 (a) Determined collision cross sections (CCS) of epoxy and dihydroxy PUFAs of different precursor fatty acids such as linoleic acid (LA), $\alpha$-linoleic acid (ALA), arachidonic acid (ARA), eicosapentaenoic acid (EPA), and docosahexaenoic acid (DHA) after derivatization with AMPP. Drift time of regioisomeric hydroxy (b), dihydroxy (c), and epoxy PUFAs (d) from ARA, EPA, and DHA after derivatization with AMPP as a function of the position of their functional group in the fatty acid carbon chain.

Shown are the drift times determined for the $\left[\mathrm{M}^{+}\right]$ion

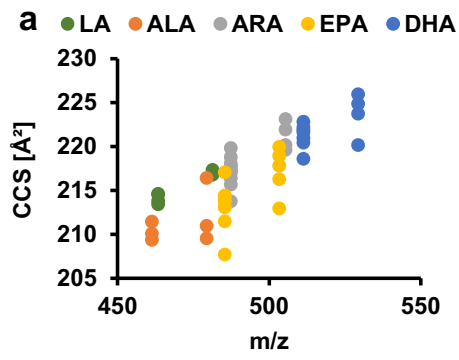

b DiHETrE DIHETE DiHDPE

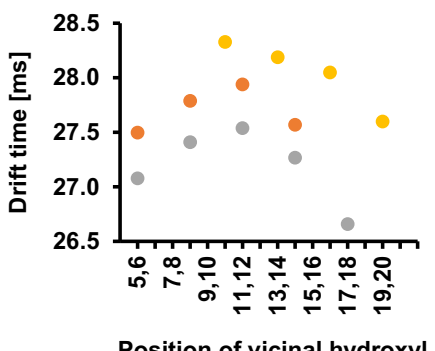

Position of vicinal hydroxyl groups
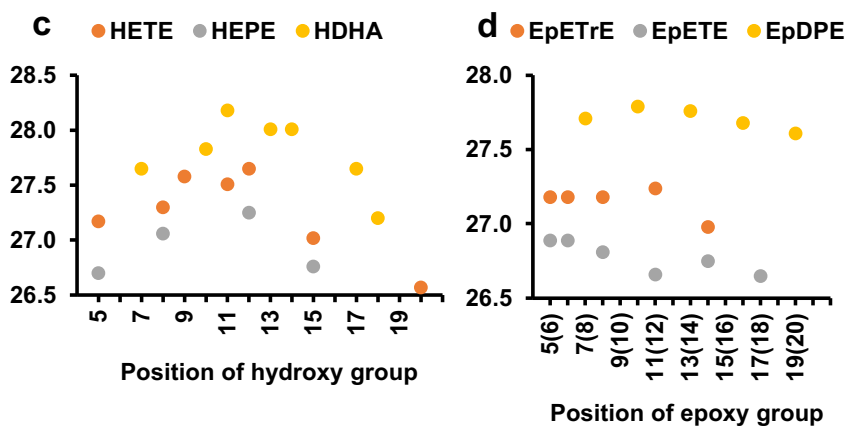

times, thereby leading to a drift time-resolved all-ion fragment spectrum (Fig. 3). Upon alternating between the fragmentation and no fragmentation mode between each IM frame, a fragment spectrum of each feature at each time step is generated. The high fragmentation mode of the LC-IM-QTOF$\mathrm{MS}(\mathrm{MS}$ ) was used to resolve co-eluting regioisomers by their specific fragmentation patterns. In contrast to QqQ methods, it was possible to differentiate between similar oxylipins, which are difficult to separate by LC or ion mobility alone [19, 54].

Figure 1 shows the fragmentation spectrum of 14(15)EpETrE-AMPP analyzed by LC-IM-QTOF-MS(/MS) in comparison with an analysis performed without derivatization on a QqQ-MS instrument. The spectrum of the derivatized analytes contains fragments from $\mathrm{m} / z, 169$ up to the $\left[\mathrm{M}^{+}\right]$ ion. The spectrum of the underivatized analyte mainly contains fragments in the $m / z>250$ range. Thus, the spectrum of the derivatized analyte contains more structural information of the analyte and offers the advantage of a better structural characterization in the frame of a non-targeted analysis. This is in agreement with the work of Yang et al., who showed that charge-switch derivatization can lead to more structural information, e.g. on the position of double bonds in fatty acids [55].

The limits of detection and quantification for the 52 derivatized analytes are comparable with recently published methods, with LODs ranging between $0.2 \mathrm{nM}$ for 12,13DiHODE and $10.0 \mathrm{nM}$ for 14,15-DiHETE [20, 22, 40, 56]. Upon alternating the high and zero fragmentation energy at 2.1 frames/s, the peak of each analyte signal is covered by more than 15 data points, allowing integration of the peak area [57]. However, a correlation between sensitivity and the type of functional group of the oxylipin could not be deduced. Hartung et al. [58] showed that in some cases, the concentration of non-certified oxylipin standards differ considerably from the nominal concentration. This could explain the
Fig. 5 Quantification of derivatized epoxy PUFAs derived from ARA in Caco-2 cells with and without incubation with TPPU for $48 \mathrm{~h}$, a soluble epoxide hydrolase inhibitor. Shown is the mean $\pm \mathrm{SD}(n=3$, ANOVA followed by Dunnett's post hoc test $* p<0.05, * * p<0.01$, $* * * p<0.001)$
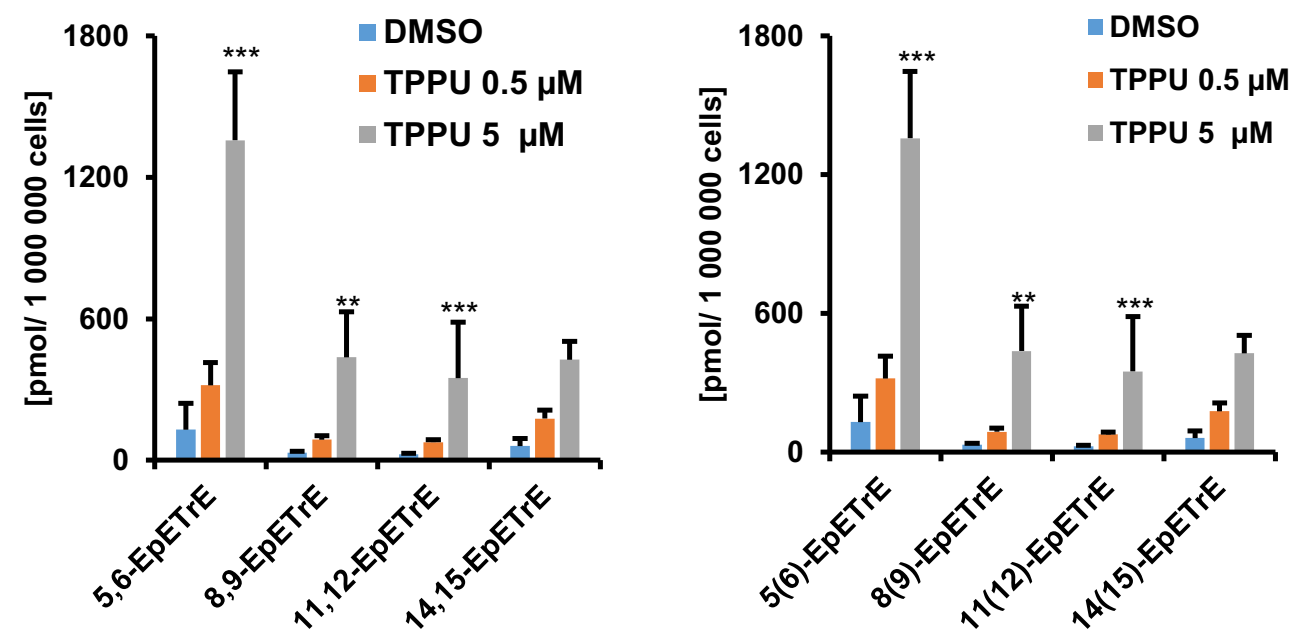

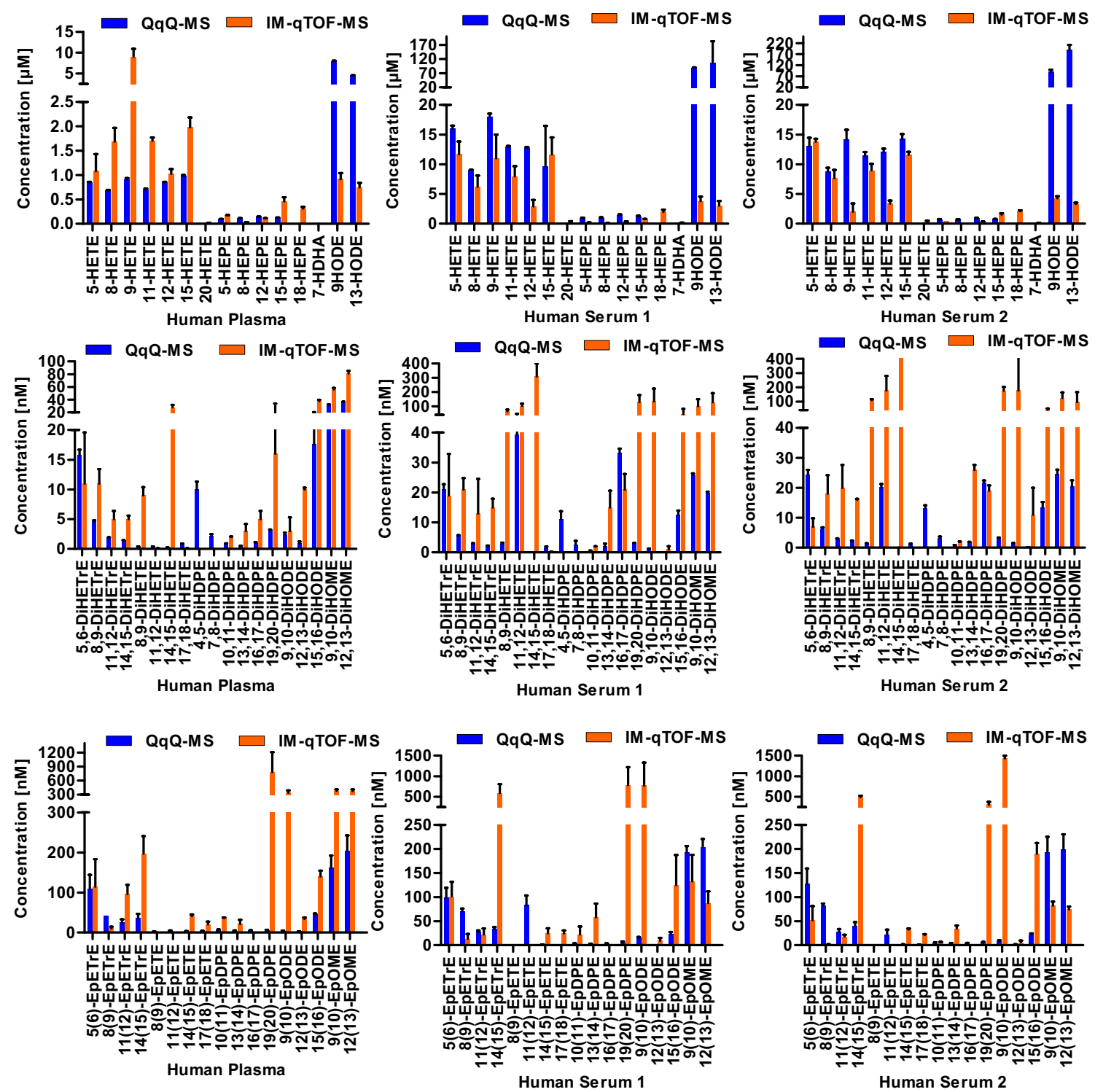

Fig. 6 Quantification of hydroxy (top), dihydroxy (middle), and epoxy PUFAs (bottom) in human plasma (right), serum 1 (middle), and serum 2 (left) using LC-IM-QTOF-MS(/MS) with derivatization with AMPP (orange) in comparison with a LC-QqQ-MS method without derivatization. Shown are the mean $\pm \operatorname{SEM}(n=3)$ diverging sensitivities described for ESI-MS-based oxylipin detection methods in the scientific literature [58]. As the standards used in the present work were not certified, this may also impact on the determined LODs and lead to the observed differences upon comparison with other methods. For most analytes, the ULOQ was $400-1000 \mathrm{nM}$, as indicated by detector saturation in the calibration runs when exceeding this concentration. This low linear range is a typical limitation of time-of-flight analyzers [59]. The IM stage results in a further accumulation of analytes due to the trapping of ions upstream of the drift tube, which leads to even more pronounced saturation effects.

The calculated CCS values of the derivatized analytes increase, as expected, with the length of the PUFA (Fig. 4a). The
CCS values from derivatized oxylipins of EPA $\left(\mathrm{C}_{20}\right)$ are in the range of 207.7-220.0 $\AA^{2}$, while the DHA $\left(\mathrm{C}_{22}\right)$ products exhibit larger CCS values of $218.6-226.0 \AA^{2}$, since the fatty acid moiety contains two additional carbon atoms. Derivatized products of ARA have slightly higher CCS values than EPA (213.8-223.1 $\AA^{2}$ ), even though both PUFAs contain 20 carbon atoms. Kyle et al. showed that monounsaturated fatty acids have higher CCS values than unsaturated fatty acids and the same correlation was shown by Zhang et al. before, with CCS increasing from C18:3 $\left(170.5 \AA^{2}\right), \mathrm{C} 18: 2$ $\left(171.3 \AA^{2}\right), \mathrm{C} 18: 1\left(172.4 \AA^{2}\right)$ to $\mathrm{C} 18: 0\left(173.7 \AA^{2}\right)[60,61]$. This correlation between the degree of saturation and the CCS value explains the deviation of CCS values between ARA and EPA, which have four and five double bonds, respectively. 


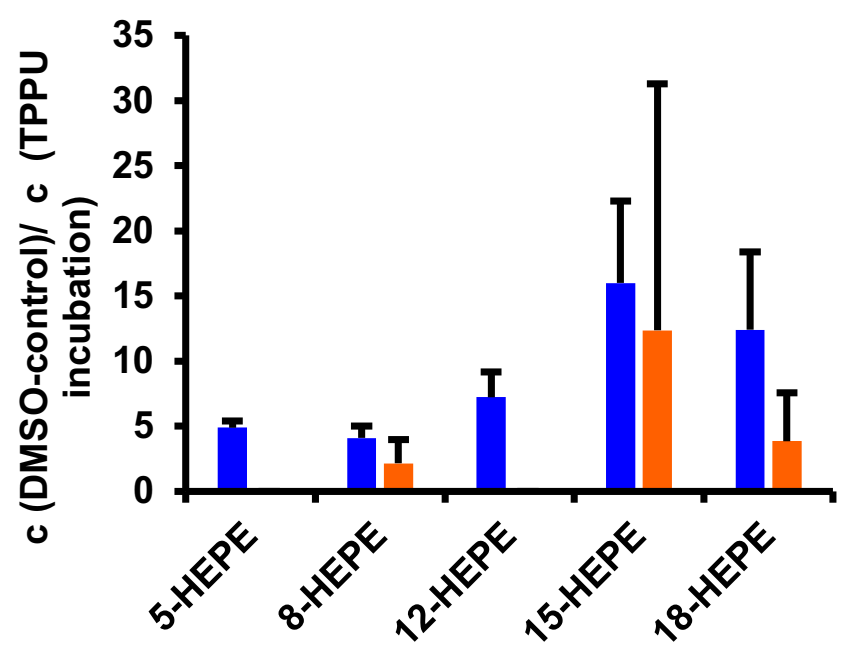

- analyzed in parallel a analyzed individually

Fig. 7 Representative relative quantification of different HEPEs in Caco2 cells with and without TPPU after ${ }^{2} \mathrm{H}_{5}$-AMPP and AMPP derivatization with parallel sample analysis using an iTRAQ-like approach in comparison with individual quantification of analytes by external calibration (mean $\pm \mathrm{SD}, n=3$ ). Missing bars were below the LLOQ for individual quantification

Kyle et al. also showed a similar correlation between CCS values and precursor fatty acids of oxylipins, e.g. values ranging from 190.2 to $199.0 \AA^{2}$ for DHA products when using nitrogen as drift gas [36]. The larger CCS values observed in the present experiments are explained by the larger molecular structure of the compounds resulting from the derivatization with AMPP. The correlation between CCS values and precursor fatty acids can be helpful for the identification of precursor fatty acids of unknown oxylipins in non-targeted approaches.

By evaluating the correlation between the CCS value and the position of the functional group for hydroxy, dihydroxy, and epoxy PUFA regioisomers, the following interesting behavior is observed (Fig. 4b): depending on the location of the functional group, the CCS value changes with a maximum between the $\mathrm{C} 10$ and the $\mathrm{C} 12$ position. This trend is explained by a smaller impact of the functional group on the CCS value when located closer to the relatively large AMPP moiety. Thus, results presented in this study suggest that the effect of the decreasing CCS when exceeding the $\mathrm{C} 10-\mathrm{C} 12$ position is caused by the interaction of the functional group with the positive charge of the AMPP derivative. This may induce ring formation, in analogy to $\mathrm{LTB}_{4}$ [35], which, in turn, would decrease the CCS. Ion mobility measurements and calculations performed by Di Giovanni et al. suggest that $\mathrm{LTB}_{4}$ is able to form a folded structure due to a stabilizing hydrogen bond between the carboxylate anion and the C5 hydroxy proton [35]. As a more compact three-dimensional ion structure caused by folding or ring formation leads to a lower CCS, the folded $\mathrm{LTB}_{4}$ had lower drift times than the open structures of
Table 2 Tentative identification of 10-hydroxy-8-octadecenoic acid by LC-IM-QTOF-MS(/MS) after derivatization with AMPP in commercially available human blood plasma samples. Shown are the corresponding feature ID, accurate mass of the precursor ion, retention time, drift time, and collision cross section $(\Omega)$ for the analyte in three identical plasma samples. Italicized numbers indicate the specific fragments of the molecule shown in Fig. S2 (see ESM)

\begin{tabular}{|c|c|c|c|}
\hline Feature ID & Plasma $1-547$ & Plasma $2-562$ & Plasma $3-565$ \\
\hline$m / z\left[\mathrm{M}^{+}\right]$ & 465.3454 & 465.3472 & 465.3462 \\
\hline Intensity & 268,569 & 232,190 & 291,112 \\
\hline Retention time (min) & 15.31 & 15.42 & 15.21 \\
\hline Drift time (ms) & 26.85 & 26.89 & 26.98 \\
\hline $\operatorname{CCS} \Omega\left(\AA^{2}\right)$ & 233.06 & 233.48 & 234.42 \\
\hline $\mathrm{m} / \mathrm{z}$ fragment 1 & - & 156.1460 & - \\
\hline $\mathrm{m} / \mathrm{z}$ fragment 2 & - & 159.0880 & - \\
\hline $\mathrm{m} / \mathrm{z}$ fragment 3 & 169.0862 & 169.0842 & 169.0881 \\
\hline$m / z$ fragment 4 & 183.0893 & 183.0882 & 183.0909 \\
\hline$m / z$ fragment 5 & 226.1078 & 226.1064 & 226.1092 \\
\hline$m / z$ fragment 6 & 239.1159 & - & 239.1170 \\
\hline$m / z$ fragment 7 & - & - & 254.1406 \\
\hline$m / z$ fragment 8 & 267.1468 & - & 267.1480 \\
\hline$m / z$ fragment 9 & - & - & 307.1808 \\
\hline $\mathrm{m} / z$ fragment 10 & 316.2620 & - & - \\
\hline $\mathrm{m} / \mathrm{z}$ fragment 11 & 335.1722 & - & 335.1754 \\
\hline $\mathrm{m} / \mathrm{z}$ fragment 12 & 352.2830 & 352.2802 & - \\
\hline $\mathrm{m} / \mathrm{z}$ fragment 13 & - & 413.3243 & - \\
\hline$m / z$ fragment 14 & - & 421.2935 & - \\
\hline $\mathrm{m} / \mathrm{z}$ fragment 15 & 423.3098 & 423.3086 & - \\
\hline $\mathrm{m} / \mathrm{z}$ fragment 16 & 435.3093 & 435.3084 & 435.3124 \\
\hline $\mathrm{m} / z$ fragment 17 & - & 451.3294 & - \\
\hline $\mathrm{m} / \mathrm{z}$ fragment 18 & 463.3313 & - & 463.3328 \\
\hline
\end{tabular}

$\mathrm{LTB}_{4}[35,62]$. With the interacting functional group located further away from the derivatized carbon acid group in the present experiments, the size of the protruding aliphatic group decreases, probably resulting in a smaller three-dimensional structure with a smaller CCS. Thus, the CCS depends on the position of the functional group within the oxylipin, leading to the smallest CCS when positioned in the mid-C-chain region.

Using the additional ion mobility separation dimension enables separation of components, which co-elute in liquid chromatography. Figure 3 shows an example of two co-eluting unidentified analytes in human plasma with the same $\mathrm{m} / \mathrm{z}$ 479.3332. In a typical non-targeted approach using LC coupled to high-resolution mass spectrometry without IM separation, these compounds cannot be detected individually. In this case, individual structure analysis is only possible by IM separation. For $\mathrm{m} / \mathrm{z}$ 479.3332, a molecular formula of $\mathrm{C}_{30} \mathrm{H}_{43} \mathrm{O}_{3} \mathrm{~N}_{2}$ is calculated, which, considering the AMPP head group, fits to a doubly oxygenated oxylipin with a $\mathrm{C}_{18}$ chain and two double bonds. This is also in agreement with 
the retention and drift times, both characteristic for shortchained dihydroxy PUFAs. A mobility-based separation of lipid isomers was also demonstrated in other studies, e.g. with phosphocholine or triacylglycerol isomers [63]. It is concluded that ion mobility proves to be of great benefit in the analysis of oxylipins in highly complex biological matrices.

\section{Quantification of oxylipins in Caco-2 cells}

The quantification of epoxy PUFAs in Caco- 2 cells showed a significant increase in the EpETrE concentration when incubated with the sEH inhibitor TPPU (Fig. 5). As expected, a lower sEH activity stabilizes epoxy PUFAs and, thus, increases their concentration [64]. Similar results were observed in numerous other studies. For instance, Ostermann et al. [65] showed a significant increase of the 14(15)-EpETrE/14,15DiHETrE ratio in plasma following oral administration of TPPU to mice. Interestingly, in other studies using Caco-2 cells, the concentration of the EpETrE remained below the LOD of $6.2 \mathrm{nM} \mathrm{[66].} \mathrm{The} \mathrm{present} \mathrm{results} \mathrm{clearly} \mathrm{demonstrate}$ the advantage of applying the developed LC-IM-QTOF-MS// MS) method in combination with charge-switch derivatization to the analysis of complex biological matrices.

\section{Quantification of oxylipins in human plasma and serum}

The quantification of oxylipins by derivatization and LC-IMQTOF-MS(/MS) is overall consistent when compared with the standard protocol using LC-QqQ-MS [21, 54]. The concentrations of analytes in the different samples are within the same order of magnitude and the relative concentrations of regioisomers are comparable (Fig. 6). The same order of magnitude for many analytes in hydrolyzed human plasma was also found by Quehenberger et al. utilizing an QqQ in ESI(-) [67]. The concentrations of most compounds analyzed with LC-IM-QTOF-MS(/MS) are slightly higher when compared with the results obtained using the LC-QqQ-MS method. As the derivatization of oxylipins with AMPP introduces a permanent positive charge in the molecule, it is less prone to ion suppression during ionization, as it already contains its charge and therefore results in higher concentrations measured with the external matrix-free calibration [38, 68]. Interestingly, some analytes, e.g. 19,20-DiHDPE, show higher concentrations compared with the LC-QqQ-MS method. The additional separation of analytes and matrix by ion mobility results in more favorable signal to noise ratios for selected analytes, a fact which could partly explain the apparent higher concentrations $[29,60]$. The detailed analysis of the observed variations in the determined concentrations in comparison with the established LC-QqQ-MS methods [7, 56] is subject to further studies. The present results show that the developed approach allows gaining biologically meaningful quantitative oxylipin patterns. The developed method allows obtaining full MS and MS/MS spectra of unknown compounds and thereby allows researchers to analyze these data retrospectively, which is not feasible with the scheduled selected reaction monitoring on a QqQ instrument.

\section{Relative quantification of oxylipins-an iTRAQ-like approach}

The concentrations determined in the parallel analysis of oxylipins in an ITRAQ analogous approach were in the same order of magnitude as concentrations of samples quantified by external calibration using internal standards. With both methods, similar ratios were found in Caco-2 cells when incubated with $5 \mu \mathrm{M}$ TPPU and compared with the DMSOtreated control group. In the case of the parallel analysis, the relative quantification was possible for more analytes, since it is not limited by the LLOQ of the external calibration. Thus, a trend to a relative increase of oxylipins was observed, which was not observed when using the external calibration (Fig. 7). While iTRAQ is usually used in proteomics analyses, we successfully transferred this method to the field of non-targeted lipidomics of oxylipins [70-72]. Lamos et al. [68] showed the advantages of isotopic labeling for the relative quantification of fatty acids in hydrolyzed egg lipid, greatly enhancing positive mode electrospray ionization sensitivity after reversedphase separation under acidic conditions. The parallel analysis of two samples omitting calibration reduces analysis time compared with individual analysis of compounds. Derivatization with ${ }^{2} \mathrm{H}_{5}$-AMPP is a simple tool to improve quantification in complex biological matrices and yields new insights, e.g. in the formation of formerly unknown oxylipins.

\section{Non-targeted analysis}

The non-targeted analysis of samples after AMPP derivatization by LC-IM-QTOF-MS(/MS) opens up new possibilities for extended data analysis. The specific cleavage of AMPP can be used to identify compounds that contain a carboxylic acid functional group such as fatty acids, oxylipins, and other eicosanoids. This improves the confidence in structure elucidation by adding a selective transition.

In the presented non-targeted workflow, including feature analysis for derivatized plasma and filtering the specific AMPP derivate fragments, about one-third of the features were derived from precursors with a carboxylic acid moiety. This information provides an initial overview of the analyzed sample. To increase the relevance of the results and to discover potentially relevant biomolecules, all three analyzed plasma samples were compared. The alternating frame mode allows such a comparison including the feature retention time, drift time, $m / z$ of the $\left[\mathrm{M}^{+}\right]$, and its fragmentation pattern. By doing so, the number of potential features from oxylipins and other 
carboxylic acids was reduced to 285 , which were consistently observed in all three human plasma samples. Due to the availability of fragment spectra for all features, it is possible to elucidate the structure of a compound of interest. For example, Fig. S2 (see ESM) and Table 2 show the tentative identification of one feature as 10-hydroxy-8-octadecenoic acid, a possible autoxidation product of oleic acid. The accurate mass of 465.3454 Da for the feature is equivalent to the calculated AMPP-derivatized oxylipin. The retention time of $15.3 \mathrm{~min}$ and the drift time of $26.9 \mathrm{~ms}$ are within the expected time windows for hydroxyl PUFAs. In addition, the observed fragment ions can be explained by the typical fragmentation pattern of hydroxy fatty acids showing an alpha cleavage next to the hydroxyl group. The observed spectra show a fragment ion at $\mathrm{m} / \mathrm{z} 352$, which is expected after the alpha cleavage of 10-hydroxy-8-octadecenoic acid. This demonstrates the potential of the developed non-targeted workflow for the structural analysis of unknown oxylipins.

In previous work, we have shown that AMPP derivatization of oxylipins does not significantly improve the analytical performance using a QqQ instrument [42], although it leads to characteristic fragmentation patterns for AMPP derivatives [42]. Poad et al. recently made use of the ozone-induced dissociation of AMPP-labeled fatty acids and the characteristic cleavage of the AMPP head group, thereby producing a fragment ion peak at $m / z 183$, to reveal the chain length and saturation degree of fatty acids [73]. The method presented in this work follows a similar direction, automating the workflow for identification of derivatized oxylipins, eicosanoids, and fatty acids in a non-targeted approach. The application of the LC-IM-QTOF-MS(/MS) method results in the advantage of a second dimension for separation based on ion mobility, which is directly related to the conformation of a molecule. This increases the specificity of the method, as unknown compounds are additionally qualified by their collision cross section.

\section{Conclusion}

Liquid chromatography in combination with ion mobility and high-resolution tandem mass spectrometry is a promising tool to enhance the effectiveness of targeted and non-targeted oxylipin analysis. In the present study, a non-targeted setup was combined with charge-switch derivatization using AMPP. The analytical performance was evaluated regarding its advantages as well as its limitations. The developed method was evaluated using 52 oxylipin standards. Limits of detection were comparable with those attainable with LC-QqQ-MS, ranging from $0.2 \mathrm{nM}$ for 12,13-DiHODE to $10 \mathrm{nM}$ for 5HETE. We analyzed pooled human plasma and two different pooled human serum samples and compared the results with a standard LC-QqQ-MS method. Despite some differences, which need to be addressed in future studies, the quantified concentrations were on average in a comparable range. This demonstrates the potential of applying the method to complex biological matrices. The biggest advantage of the presented workflow is the non-targeted analysis of unknown features. The combination of AMPP derivatization, LC-IM-QTOFMS(/MS) analysis, and computer-aided data analysis facilitates the identification of unknown analytes via the specific cleavage of the AMPP. This workflow enabled us to consistently detect 285 features in human blood samples and, therefore, can be used to analyze complex biological samples to find new oxylipins involved in biological processes.

Acknowledgments The authors would like to thank Laura Menke for excellent technical assistance in the performance of the cell culture experiments.

Funding information Open Access funding provided by Projekt DEAL. This work was in part supported by the German Research Foundation (DFG, grant Sche 1801).

\section{Compliance with ethical standards}

Conflict of interest The authors declare that they have no conflict of interest

Open Access This article is licensed under a Creative Commons Attribution 4.0 International License, which permits use, sharing, adaptation, distribution and reproduction in any medium or format, as long as you give appropriate credit to the original author(s) and the source, provide a link to the Creative Commons licence, and indicate if changes were made. The images or other third party material in this article are included in the article's Creative Commons licence, unless indicated otherwise in a credit line to the material. If material is not included in the article's Creative Commons licence and your intended use is not permitted by statutory regulation or exceeds the permitted use, you will need to obtain permission directly from the copyright holder. To view a copy of this licence, visit http://creativecommons.org/licenses/by/4.0/.

\section{References}

1. Buczynski MW, Dumlao DS, Dennis EA. Thematic review series: proteomics. An integrated omics analysis of eicosanoid biology. J Lipid Res. 2009;50(6):1015-38.

2. Funk CD. Prostaglandins and leukotrienes: advances in eicosanoid biology. Science. 2001;294(5548):1871-5.

3. Serhan CN, Chiang N, van Dyke TE. Resolving inflammation: dual anti-inflammatory and pro-resolution lipid mediators. Nat Rev Immunol. 2008;8(5):349-61.

4. Willenberg I, Rund K, Rong S, Shushakova N, Gueler F, Schebb $\mathrm{NH}$. Characterization of changes in plasma and tissue oxylipin levels in LPS and CLP induced murine sepsis. Inflamm Res. 2016;65(2):133-42.

5. Willenberg I, Ostermann AI, Schebb NH. Targeted metabolomics of the arachidonic acid cascade: current state and challenges of LC-MS analysis of oxylipins. Anal Bioanal Chem. 2015;407(10):2675-83.

6. Dumlao DS, Buczynski MW, Norris PC, Harkewicz R, Dennis EA. High-throughput lipidomic analysis of fatty acid derived 
eicosanoids and $\mathrm{N}$-acylethanolamines. Biochim Biophys Acta. 2011;1811(11):724-36.

7. Gladine C, Ostermann AI, Newman JW, Schebb NH. MS-based targeted metabolomics of eicosanoids and other oxylipins: analytical and inter-individual variabilities. Free Radic Biol Med. 2019;144:72-89.

8. Mosblech A, Feussner I, Heilmann I. Oxylipins: structurally diverse metabolites from fatty acid oxidation. Plant Physiol Biochem. 2009;47(6):511-7.

9. Serhan $\mathrm{CN}$. Novel lipid mediators and resolution mechanisms in acute inflammation: to resolve or not? Am J Pathol. 2010;177(4): 1576-91.

10. Morisseau C, Inceoglu B, Schmelzer K, Tsai H-J, Jinks SL, Hegedus CM, et al. Naturally occurring monoepoxides of eicosapentaenoic acid and docosahexaenoic acid are bioactive antihyperalgesic lipids. J Lipid Res. 2010;51(12):3481-90.

11. Newman JW, Morisseau C, Hammock BD. Epoxide hydrolases: their roles and interactions with lipid metabolism. Prog Lipid Res. 2005;44(1):1-51.

12. Smith WL, Murphy RC. The eicosanoids. In: Ridgway N, McLeod $\mathrm{R}$, editors. Biochemistry of lipids, lipoproteins and membranes. Sixth ed. Amsterdam: Elsevier; 2016. p. 259-96.

13. Archambault A-S, Turcotte C, Martin C, Provost V, Larose M-C, Laprise C, et al. Comparison of eight 15-lipoxygenase (LO) inhibitors on the biosynthesis of 15-LO metabolites by human neutrophils and eosinophils. PLoS One. 2018;13(8):e0202424.

14. Henderson WR, Harley JB, Fauci AS. Arachidonic acid metabolism in normal and hypereosinophilic syndrome human eosinophils: generation of leukotrienes B4, C4, D4 and 15-lipoxygenase products. Immunology. 1984;51(4):679-86.

15. Feltenmark S, Gautam N, Brunnström A, Griffiths W, Backman L, Edenius C, et al. Eoxins are proinflammatory arachidonic acid metabolites produced via the 15-lipoxygenase-1 pathway in human eosinophils and mast cells. Proc Natl Acad Sci U S A. 2008;105(2):680-5.

16. Fischer R, Konkel A, Mehling H, Blossey K, Gapelyuk A, Wessel $\mathrm{N}$, et al. Dietary omega- 3 fatty acids modulate the eicosanoid profile in man primarily via the CYP-epoxygenase pathway. J Lipid Res. 2014;55(6):1150-64.

17. Arnold C, Markovic M, Blossey K, Wallukat G, Fischer R, Dechend $\mathrm{R}$, et al. Arachidonic acid-metabolizing cytochrome P450 enzymes are targets of \{omega $\}-3$ fatty acids. J Biol Chem. 2010;285(43):32720-33.

18. Capdevila JH, Falck JR. Biochemical and molecular properties of the cytochrome P450 arachidonic acid monooxygenases. Prostaglandins Other Lipid Mediat. 2002;68-69:325-44.

19. Yang J, Schmelzer K, Georgi K, Hammock BD. Quantitative profiling method for oxylipin metabolome by liquid chromatography electrospray ionization tandem mass spectrometry. Anal Chem. 2009;81(19):8085-93.

20. Strassburg K, Huijbrechts AML, Kortekaas KA, Lindeman JH, Pedersen TL, Dane A, et al. Quantitative profiling of oxylipins through comprehensive LC-MS/MS analysis: application in cardiac surgery. Anal Bioanal Chem. 2012;404(5):1413-26.

21. Ostermann AI, Willenberg I, Schebb NH. Comparison of sample preparation methods for the quantitative analysis of eicosanoids and other oxylipins in plasma by means of LC-MS/MS. Anal Bioanal Chem. 2015;407(5):1403-14.

22. Rund KM, Ostermann AI, Kutzner L, Galano J-M, Oger C, Vigor C, et al. Development of an LC-ESI(-)-MS/MS method for the simultaneous quantification of 35 isoprostanes and isofurans derived from the major n3- and n6-PUFAs. Anal Chim Acta. 2018;1037:63-74.

23. Yuan Z-X, Majchrzak-Hong S, Keyes GS, Iadarola MJ, Mannes AJ, Ramsden CE. Lipidomic profiling of targeted oxylipins with ultra-performance liquid chromatography-tandem mass spectrometry. Anal Bioanal Chem. 2018;410(23):6009-29.
24. Thakare R, Chhonker YS, Gautam N, Nelson A, Casaburi R, Criner $\mathrm{G}$, et al. Simultaneous LC-MS/MS analysis of eicosanoids and related metabolites in human serum, sputum and BALF. Biomed Chromatogr. 2018;32(3):e4102.

25. Puppolo M, Varma D, Jansen SA. A review of analytical methods for eicosanoids in brain tissue. J Chromatogr B Analyt Technol Biomed Life Sci. 2014;964:50-64.

26. Kingsley PJ, Marnett LJ. LC-MS-MS analysis of neutral eicosanoids. In: Brown HA, editor. Lipidomics and bioactive lipids. Specialized analytical methods and lipids in disease, vol. 433. Amsterdam, Boston: Elsevier/Academic Press; 2007. p. 91-112.

27. Chocholoušková M, Jirásko R, Vrána D, Gatěk J, Melichar B, Holčapek M. Reversed phase UHPLC/ESI-MS determination of oxylipins in human plasma: a case study of female breast cancer. Anal Bioanal Chem. 2019;411(6):1239-51.

28. Wang Y, Armando AM, Quehenberger O, Yan C, Dennis EA. Comprehensive ultra-performance liquid chromatographic separation and mass spectrometric analysis of eicosanoid metabolites in human samples. J Chromatogr A. 2014;1359:60-9.

29. Paglia G, Kliman M, Claude E, Geromanos S, Astarita G. Applications of ion-mobility mass spectrometry for lipid analysis. Anal Bioanal Chem. 2015;407(17):4995-5007.

30. Hinz C, Liggi S, Mocciaro G, Jung SM, Induruwa I, Pereira MCDA, et al. A comprehensive UHPLC ion mobility QTOF method for profiling and quantification of eicosanoids, other oxylipins and fatty acids. Anal Chem. 2019;91(13):8025-35.

31. Hines KM, May JC, McLean JA, Xu L. Evaluation of collision cross section calibrants for structural analysis of lipids by traveling wave ion mobility-mass spectrometry. Anal Chem. 2016;88(14):7329-36.

32. Valentine SJ, Kulchania M, Barnes CAS, Clemmer DE. Multidimensional separations of complex peptide mixtures: a combined high-performance liquid chromatography/ion mobility/timeof-flight mass spectrometry approach. Int J Mass Spectrom. 2001;212(1-3):97-109.

33. Williamson LN, Bartlett MG. Quantitative liquid chromatography/ time-of-flight mass spectrometry. Biomed Chromatogr. 2007;21(6): 567-76.

34. Paglia G, Angel P, Williams JP, Richardson K, Olivos HJ, Thompson JW, et al. Ion mobility-derived collision cross section as an additional measure for lipid fingerprinting and identification. Anal Chem. 2015;87(2):1137-44.

35. Di Giovanni JP, Barkley RM, Jones DNM, Hankin JA, Murphy RC. Tandem mass spectrometry and ion mobility reveals structural insight into eicosanoid product ion formation. J Am Soc Mass Spectrom. 2018;29(6):1231-41.

36. Kyle JE, Aly N, Zheng X, Burnum-Johnson KE, Smith RD, Baker ES. Evaluating lipid mediator structural complexity using ion mobility spectrometry combined with mass spectrometry. Bioanalysis. 2018;10(5):279-89.

37. Bollinger JG, Thompson W, Lai Y, Oslund RC, Hallstrand TS, Sadilek M, et al. Improved sensitivity mass spectrometric detection of eicosanoids by charge reversal derivatization. Anal Chem. 2010;82(16):6790-6.

38. Gosetti F, Mazzucco E, Zampieri D, Gennaro MC. Signal suppression/enhancement in high-performance liquid chromatography tandem mass spectrometry. J Chromatogr A. 2010;1217(25): 3929-37.

39. Frankfater C, Jiang X, Hsu F-F. Characterization of long-chain fatty acid as $\mathrm{N}$-(4-aminomethylphenyl) pyridinium derivative by MALDI LIFT-TOF/TOF mass spectrometry. J Am Soc Mass Spectrom. 2018;29(8):1688-99.

40. Giménez-Bastida JA, Shibata T, Uchida K, Schneider C. Roles of 5lipoxygenase and cyclooxygenase- 2 in the biosynthesis of hemiketals E2 and D2 by activated human leukocytes. FASEB J. 2017;31(5):1867-78. 
41. Kutzner L, Rund KM, Ostermann AI, Hartung NM, Galano J-M, Balas L, et al. Development of an optimized LC-MS method for the detection of specialized pro-resolving mediators in biological samples. Front Pharmacol. 2019;10(169):1-19.

42. Meckelmann SW, Hellhake S, Steuck M, Krohn M, Schebb NH. Comparison of derivatization/ionization techniques for liquid chromatography tandem mass spectrometry analysis of oxylipins. Prostaglandins Other Lipid Mediat. 2017;130:8-15.

43. Empl MT, Cai H, Wang S, Junginger J, Kostka T, HewickerTrautwein M, et al. Effects of a grapevine shoot extract containing resveratrol and resveratrol oligomers on intestinal adenoma development in mice: in vitro and in vivo studies. Mol Nutr Food Res. 2018;62(2):1700450.

44. Ostermann AI, Koch E, Rund KM, Kutzner L, Mainka M, Schebb NH. Targeting esterified oxylipins by LC-MS - effect of sample preparation on oxylipin pattern. Prostaglandins Other Lipid Mediat. 2020;146:106384.

45. Henderson CM, Shulman NJ, MacLean B, MacCoss MJ, Hoofnagle AN. Skyline performs as well as vendor software in the quantitative analysis of serum 25 -hydroxy vitamin D and vitamin D binding globulin. Clin Chem. 2018;64(2):408-10.

46. Pino LK, Searle BC, Bollinger JG, Nunn B, MacLean B, MacCoss MJ. The skyline ecosystem: informatics for quantitative mass spectrometry proteomics. Mass Spectrom Rev. 2017;3(39):229-44.

47. Schilling B, Rardin MJ, MacLean BX, Zawadzka AM, Frewen BE, Cusack MP, et al. Platform-independent and label-free quantitation of proteomic data using MS1 extracted ion chromatograms in skyline: application to protein acetylation and phosphorylation. Mol Cell Proteomics. 2012;11(5):202-14.

48. Murphy RC, Barkley RM, Zemski Berry K, Hankin J, Harrison K, Johnson $\mathrm{C}$, et al. Electrospray ionization and tandem mass spectrometry of eicosanoids. Anal Biochem. 2005;346(1):1-42.

49. Morgan AH, Hammond VJ, Morgan L, Thomas CP, Tallman KA, Garcia-Diaz YR, et al. Quantitative assays for esterified oxylipins generated by immune cells. Nat Protoc. 2010;5(12):1919-31.

50. Roth KDW, Huang Z-H, Sadagopan N, Watson JT. Charge derivatization of peptides for analysis by mass spectrometry. Mass Spectrom Rev. 1998;17(4):255-74.

51. Miyashita M, Hanai Y, Awane H, Yoshikawa T, Miyagawa H. Improving peptide fragmentation by $\mathrm{N}$-terminal derivatization with high proton affinity. Rapid Commun Mass Spectrom. 2011;25(9): $1130-40$.

52. Lingeman H, Underberg WJM, editors. Detection-oriented derivatization techniques in liquid chromatography. Chromatographic science series, vol. 48. New York: Dekker; 1990.

53. Neue UD, Tran K, Méndez A, Carr PW. The combined effect of silanols and the reversed-phase ligand on the retention of positively charged analytes. J Chromatogr A. 2005;1063(1-2):35-45.

54. Ostermann AI, Willenberg I, Weylandt KH, Schebb NH. Development of an online-SPE-LC-MS/MS method for 26 hydroxylated polyunsaturated fatty acids as rapid targeted metabolomics approach for the LOX, CYP, and autoxidation pathways of the arachidonic acid cascade. Chromatographia. 2015;78(5-6):415-28.

55. Yang K, Dilthey BG, Gross RW. Identification and quantitation of fatty acid double bond positional isomers: a shotgun lipidomics approach using charge-switch derivatization. Anal Chem. 2013;85(20):9742-50.

56. Zhang X, Yang N, Ai D, Zhu Y. Systematic metabolomic analysis of eicosanoids after omega-3 polyunsaturated fatty acid supplementation by a highly specific liquid chromatography-tandem mass spectrometry-based method. J Proteome Res. 2015;14(4):1843-53.

57. Dyson N. Peak distortion, data sampling errors and the integrator in the measurement of very narrow chromatographic peaks. J Chromatogr A. 1999;842(1-2):321-40.

58. Hartung NM, Mainka M, Kampschulte N, Ostermann AI, Schebb NH. A strategy for validating concentrations of oxylipin standards for external calibration. Prostaglandins Other Lipid Mediat. 2019;141:22-4.
59. Rousu T, Tolonen A. Comparison of unit resolution SRM and TOFMS at 12,000 mass resolution for quantitative bioanalysis of 11 steroids from human plasma. Bioanalysis. 2012;4(5):555-63.

60. Kyle JE, Zhang X, Weitz KK, Monroe ME, Ibrahim YM, Moore $\mathrm{RJ}$, et al. Uncovering biologically significant lipid isomers with liquid chromatography, ion mobility spectrometry and mass spectrometry. Analyst. 2016;141(5):1649-59.

61. Zhang F, Guo S, Zhang M, Zhang Z, Guo Y. Characterizing ion mobility and collision cross section of fatty acids using electrospray ion mobility mass spectrometry. J Mass Spectrom. 2015;50(7):90613.

62. Shvartsburg AA, Hudgins RR, Dugourd P, Jarrold MF. Structural elucidation of fullerene dimers by high-resolution ion mobility measurements and trajectory calculation simulations. J Phys Chem A. 1997;101(9):1684-8.

63. Bowman AP, Abzalimov RR, Shvartsburg AA. Broad separation of isomeric lipids by high-resolution differential ion mobility spectrometry with tandem mass spectrometry. J Am Soc Mass Spectrom. 2017;28(8):1552-61.

64. Morisseau C, Hammock BD. Epoxide hydrolases: mechanisms, inhibitor designs, and biological roles. Annu Rev Pharmacol Toxicol. 2005;45:311-33.

65. Ostermann AI, Herbers J, Willenberg I, Chen R, Hwang SH, Greite R, Morisseau C, Gueler F, Hammock BD, Schebb NH (2015) Oral treatment of rodents with soluble epoxide hydrolase inhibitor 1-(1propanoylpiperidin-4-yl)-3-4-(trifluoromethoxy)phenylurea (TPPU): Resulting drug levels and modulation of oxylipin pattern. Prostaglandins Other Lipid Mediat 121:131-37.

66. Le Faouder P, Baillif V, Spreadbury I, Motta J-P, Rousset P, Chêne $\mathrm{G}$, et al. LC-MS/MS method for rapid and concomitant quantification of pro-inflammatory and pro-resolving polyunsaturated fatty acid metabolites. J Chromatogr B Analyt Technol Biomed Life Sci. 2013;932:123-33.

67. Quehenberger O, Dahlberg-Wright S, Jiang J, Armando AM, Dennis EA. Quantitative determination of esterified eicosanoids and related oxygenated metabolites after base hydrolysis. J Lipid Res. 2018;59(12):2436-45.

68. Lamos SM, Shortreed MR, Frey BL, Belshaw PJ, Smith LM (2007) Relative quantification of carboxylic acid metabolites by liquid chromatography-mass spectrometry using isotopic variants of cholamine. Anal Chem 79(14):5143-49.

69. Remane D, Meyer MR, Wissenbach DK, Maurer HH. Ion suppression and enhancement effects of co-eluting analytes in multi-analyte approaches: systematic investigation using ultra-high-performance liquid chromatography/mass spectrometry with atmosphericpressure chemical ionization or electrospray ionization. Rapid Commun Mass Spectrom. 2010;24(21):3103-8.

70. Aggarwal K, Choe LH, Lee KH. Shotgun proteomics using the iTRAQ isobaric tags. Brief Funct Genomic Proteomic. 2006;5(2): 112-20.

71. Cong Y-S, Fan E, Wang E. Simultaneous proteomic profiling of four different growth states of human fibroblasts, using aminereactive isobaric tagging reagents and tandem mass spectrometry. Mech Ageing Dev. 2006;127(4):332-43.

72. Hardt M, Witkowska HE, Webb S, Thomas LR, Dixon SE, Hall SC, et al. Assessing the effects of diurnal variation on the composition of human parotid saliva: quantitative analysis of native peptides using iTRAQ reagents. Anal Chem. 2005;77(15):4947-54.

73. Poad BLJ, Marshall DL, Harazim E, Gupta R, Narreddula VR, Young RSE, et al. Combining charge-switch derivatization with ozone-induced dissociation for fatty acid analysis. J Am Soc Mass Spectrom. 2019;30(10):2135-43.

Publisher's note Springer Nature remains neutral with regard to jurisdictional claims in published maps and institutional affiliations. 